\title{
Evaluation of Sustainable Structural Concrete Using Recycled Aggregate and Aramid Fiber Sheet
}

\author{
Y. S. Cho, ${ }^{1}$ H. S. Jang, ${ }^{1}$ S. K. Back, ${ }^{1}$ M. I. Choi, ${ }^{1}$ S. U. Hong, ${ }^{2}$ and Y. T. Lee ${ }^{2}$ \\ ${ }^{1}$ Division of Architecture and Architectural Engineering, Hanyang University, 55 Hanyangdaehakro, Sa-1-dong, \\ Sangnok-gu, Kyungki-do 426-791, Republic of Korea \\ ${ }^{2}$ Hanbat National University, Yuseong-gu, Daejeon 305-719, Republic of Korea
}

Correspondence should be addressed to Y. S. Cho; ycho@hanyang.ac.kr

Received 16 May 2016; Accepted 5 October 2016

Academic Editor: Cristina Leonelli

Copyright (C) 2016 Y. S. Cho et al. This is an open access article distributed under the Creative Commons Attribution License, which permits unrestricted use, distribution, and reproduction in any medium, provided the original work is properly cited.

\begin{abstract}
The purpose of this study was to evaluate the flexural performance of recycled aggregate RC beam reinforced with aramid fiber sheets. Compressive strength of concrete using recycled aggregate is generally similar or slightly lower than normal concrete. To improve the compressive strength, aramid fiber sheets have been used in this study. This study examines the structural behavior of concrete beams prepared with recycled aggregate and strengthened aramid fiber sheets at varying locations. One concrete beam as a control specimen, that is prepared with 30 percent recycled aggregate and 70 percent natural aggregate, has been tested, and 3 more strengthened beams (bottom, bottom and sides, bottom and both ends with U-shaped strengthened beams) are tested. The ultimate loads have increased by $38.01 \%, 39.88 \%$, and $100.79 \%$ for bottom, bottom and sides, bottom and both ends with Ushaped strengthened beams. The ductility ratios are $2.75 \sim 6.20$ for strengthened beams. The experimental results showed that the strengthening system with U-shaped band controls the premature debonding and provides a more ductile failure mode than the strengthening system without U-shaped bands. It can be found that the ultimate strength of H40-RGA30-BS specimen based on load-deflection curves shows most promising result. The experimental results are compared with the analytical results of nonlinear flexural behaviors for strengthened reinforced recycled aggregate concrete beam.
\end{abstract}

\section{Introduction}

Reinforced concrete structure may decrease in structural resistance due to installation factor, aging by lapse of time and inappropriate maintenance, and so forth, and it sometimes causes many problems regarding structure safety. A structure that decreased in resistance due to various combined causes requires swift repair and reinforcement, and an effective method to reinforce such reinforced concrete structure is the method of attaching external reinforcing plate where steel plate or fiber sheet is reinforced with epoxy, but only very limited data are available for this method. Steel plate attaching method allows barely any change in the cross section of member and enables simple installation, but this method is inconvenient to handle and vulnerable to corrosion, offers weak fire-resistance performance, and demonstrates brittle spalling of reinforcement plate. Fiber reinforcement materials like glass, carbon, and aramid [1] are drawing attentions due to their various advantages such as easy production and simple handling in addition to high strength, light weight, corrosion-resistance, chemicalresistance, and fatigue-resistance. These materials contribute to promotion of structure resistance, but due to their linear material characteristics, reinforcement method, and epoxy material characteristics, it is difficult to define their effects of reinforcement clearly [2]. Also, recently owing to reinforcement of quality standard for recycled aggregates and steady technical development of construction wastes companies, high class treated recycled aggregates [3] are in production. In tune with such trend, the quality of recycled aggregates [4] is being evaluated and studied in various aspects, and it is known that recycled aggregates demonstrate material performances that approximate those of natural aggregates. Therefore, the purpose of this study is to develop sustainable structural concrete [5] by evaluating the flexural performance of RC beam to which recycled aggregates are substituted 
by $30 \%$ and by applying aramid fiber sheets by specifying the $\mathrm{RC}$ beam as reference specimen in order to provide comprehensive data for recycled aggregates and alternative resource to natural aggregates which are decreasing in reserve and to acquire reliability of it.

In order to identify the flexural characteristics of beam reinforced with aramid fiber sheets, 4 RC beams to which recycled aggregates were mixed were produced, and after applying unreinforced specimen, specimen reinforced at the bottom part, specimen reinforced at the bottom part and both end parts, and specimen reinforced at the bottom part and side part, respectively, the flexural performance of aramid fiber sheet RC beam was identified through analysis of the load-bending curve, concrete crack and fiber sheet rupture pattern, yield strength, maximum load, and ductility index measured in the experiment. Also, in order to verify the validity of the reinforced beam experiment, the results of flexural experiment and the theoretical analysis results were compared [6].

Reinforced concrete (RC) beams may be strengthened for shear using externally bonded fiber reinforced polymer (FRP) composites in the form of side bonding, $\mathrm{U}$-jacketing, or complete wrapping [7]. The shear failure of almost all RC beams shear-strengthened with side bonded FRP and the majority of those strengthened with FRP U-jackets is due to debonding of the FRP. The bond behavior between the externally bonded FRP reinforcement (referred to as FRP strips for simplicity) and the concrete substrate therefore plays a crucial role in the failure process of these beams. The validity of the analytical solution is verified by comparing its predictions with numerical results from a finite element analysis [8]. This analytical treatment represents a significant step forward in understanding how interaction between FRP strips, steel stirrups, and concrete affects the shear resistance of RC beams shear strengthened with FRP strips. Aramid fiber composite rectangular tendons have potential as reinforcements for concrete structures [9]. Recent studies on prestressed concrete beams have showed brittle flexural failure due to the elastic rupture of the FRPs; however, for the maintenance and hazard anticipation of structures, the brittle failure mode is undesirable. In order to improve the ductility, a series of flexural tests was carried out on beams with bonded and/or unbonded rectangular rebars or with additional nontensioned, regular reinforced rebars. Test results showed that ultimate deformation in the beam with unbonded rectangular rebars was $250 \%$ that of the beam with bonded rectangular rebars, while the loading capacity was $85 \%$. The additional placement of reinforced rectangular rebars produced a $450 \%$ higher ultimate deformation. It was found that the ductility was significantly enhanced by the use of combination of bonded and unbounded rectangular rebars.

1.1. Study Scope and Method. In order to evaluate the flexural performance of recycled aggregates RC beam, three types of beams reinforced with aramid fiber sheets and 4 types of recycled aggregate RC beam specimens not reinforced

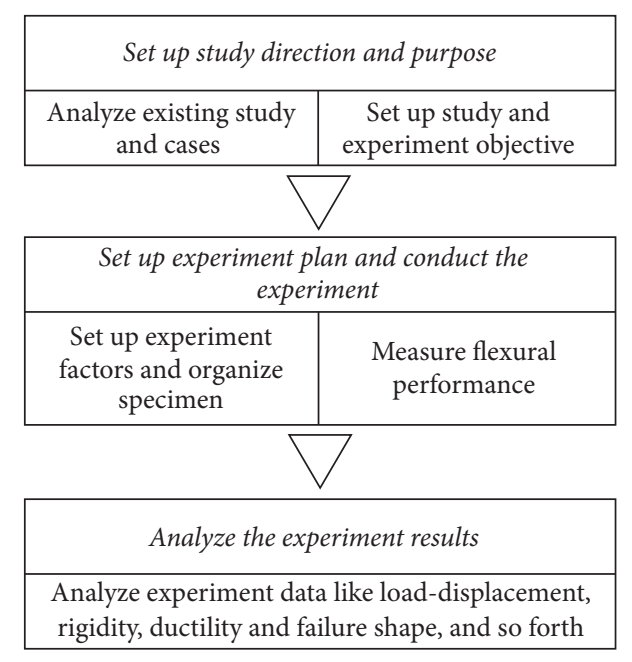

FIgURE 1: The overall scope and flow of study.

were produced, and through experiment, the flexural performances by each type were compared and analyzed. Figure 1 shows the arrangement of the overall study flow.

\section{Theoretical Background}

2.1. Sheet Tensile Rupture Load $\left(P_{t}\right)$. If sheet tensile strength reaches extreme level in flexurally reinforced beam [10], the sheet begins to tear off from the point where its tensile strength reached limit. The load at such moment is predicted in ultimate strength design method. The strain diagram and stress diagram of the beam were assumed as shown in Figure 2 [11].

At this time, the sheet was assumed to behave as one body until rupture. The strain, $\epsilon_{f}$, upon breaking of fiber sheet, where it was assumed that Hook's law applies until immediately before breaking, was obtained by dividing the tensile strength, $f_{f}$, of fiber sheet by Young's modulus, $E_{f}$, as shown in the following equation:

$$
\epsilon_{f}=\frac{f_{f}}{E_{f}} .
$$

Neutral axis distance $x$ was obtained from the balance condition of resistance. Here the resistances of concrete, compressive rebar, tensile rebar, and fiber sheet can be expressed as follows:

$$
\begin{aligned}
& C_{c}=0.85 f_{c k} b \beta_{1} x, \\
& C_{s}=\left(E_{s} \epsilon_{s}^{\prime}-0.85 f_{c k}\right) A_{s}^{\prime}, \\
& T_{s}=f_{y} A_{s}, \\
& T_{s}=f_{f} A_{f}, \\
& \beta_{1}=0.85-\left(f_{c k}-28\right) * 0.007 \geq 0.65,
\end{aligned}
$$




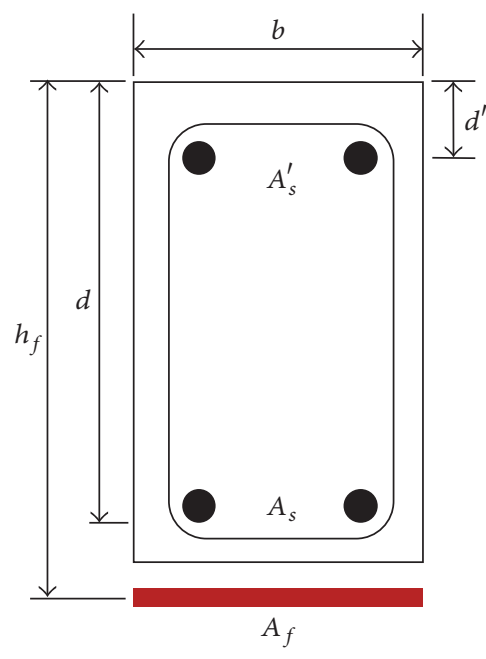

(a) Cross section

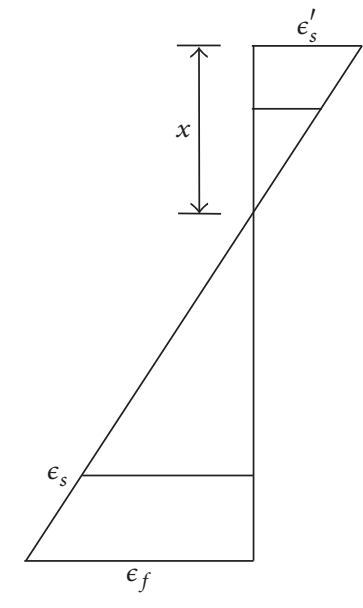

(b) Strain diagram

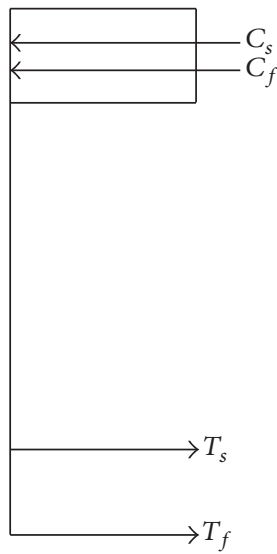

(c) Stress diagram

FIGURE 2: The cross section and stress-strain diagram of the beam.

Here, the strain of compressive rebar [12], $\epsilon_{s}^{\prime}$, is as shown in the following equation in linear strain diagram:

$$
\epsilon_{s}^{\prime}=\frac{x-d^{\prime}}{h_{f}-x} \epsilon_{f} .
$$

Neutral axis distance $x$ is as shown in the following equation:

$$
x=\frac{B-\sqrt{B^{2}-4 A C}}{2 A},
$$

where

$$
\begin{aligned}
& A=0.85 f_{c k} b \beta_{1}, \\
& B=A * h_{f}+E_{s} A_{s}^{\prime} \epsilon_{f}+0.85 f_{c k} A_{s}^{\prime}+f_{y} A_{s}+f_{f} A_{f}, \\
& C=E_{s}+E_{s} A_{s}^{\prime} \epsilon_{f} d^{\prime}\left(0.85 f_{c k} A_{s}^{\prime}+f_{y} A_{s}+f_{f} A_{f}\right) h_{f} .
\end{aligned}
$$

$h_{f}$ indicates the distance from the compressive end of the beam to the center of fiber sheet. If the neutral axis distance and resistance are obtained and substituted into (6), the tensile failure moment of sheet, $M_{t}$, can be obtained:

$$
\begin{aligned}
M_{t}= & C_{e}\left(d-\frac{\beta_{1} x}{2}\right)+C_{s}\left(x-d^{\prime}\right)+T_{s}(d-x) \\
& +T_{f}\left(h_{f}-x\right) .
\end{aligned}
$$

Therefore, in case trisection point loading method is applied to simply supported beam, the tensile rupture load of sheet is as in the following equation:

$$
P_{t}=\frac{3 M_{t}}{L} \text {. }
$$

" $L$ " indicates the distance between the locations of beam.
2.2. Sheet End Rip-Off Load $\left(P_{s}\right)$. If the stress concentrated on sheet end exceeds the adhering strength of epoxy, sheet end may rip off, and if the load at this time is obtained with the use of approximation solution of Roberts [13], the shear stress, $\tau$, of sheet end can be expressed as follows:

$$
\tau=V+\left[\frac{K_{s}}{E_{f} b_{f} d_{f}}\right]^{1 / 2} M \frac{b_{f} d_{f}}{I b_{a}}\left(h_{f}-x\right) .
$$

$b_{f}, d_{f}$, and $E_{f}$ indicate the width, thickness, and Young's modulus, respectively, and $b_{a}$ and $K_{s}$ are the shear strengths of epoxy per width and length, respectively. Also, shear force $V$ is expressed as in equation (9), and moment $M$ is expressed as in equation (10):

$$
\begin{aligned}
V & =P, \\
M & =P a .
\end{aligned}
$$

$a$ is expressed as in the following equation at a location apart by $\left(h+d_{f}\right) / 2$ from sheet end to the center:

$$
a=\frac{L-L_{f}}{2}+\frac{h_{f}-d_{f}}{2} .
$$

If equations (9) and (10) are substituted into (8), shear stress $\tau$ can be arranged as follows:

$$
\tau=P+\left[\frac{K_{s}}{E_{f} b_{f} d_{f}}\right]^{1 / 2} P_{a} \frac{b_{f} d_{f}}{I b_{a}}\left(h_{f}-x\right) .
$$

If the above expression is substituted to load $P$ and arranged, end rip-off load $P_{s}$ can be expressed as follows:

$$
P_{s}=\frac{2 \tau}{1+\left[K_{s} / E_{f} b_{f} d_{f}\right]^{1 / 2} a\left(b_{f} d_{f} / I b_{a}\right)\left(h_{f}-x\right)} .
$$




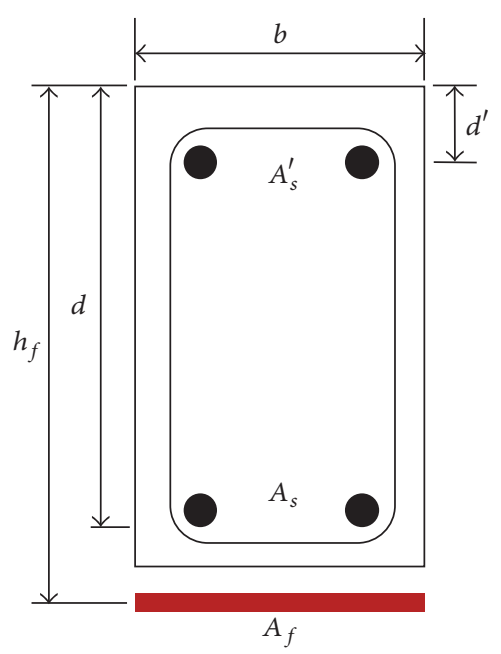

(a) Cross section

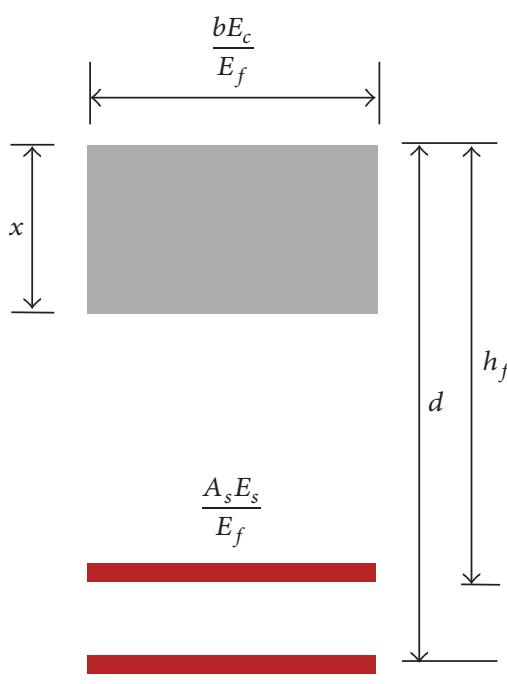

(b) Converted cross section

FIgURE 3: The converted cross section for fiber sheets.

$K_{s}$ indicates shear strength per the unit length of epoxy and can be expressed as in the following equation:

$$
K_{s}=G_{s} \frac{b_{a}}{d_{a}},
$$

where $G_{s}$ and $d_{a}$ indicate the shear coefficient and thickness of epoxy resin, respectively. In order to obtain neutral axis distance $x$ and cross section secondary moment $I$, the sheets were assumed to be in complete junction. As shown in Figure 3(b), the neutral axis distance obtained with the use of the cross section that converted the compressive side cross section of concrete and the cross section of tensile and compressive rebar to fiber sheets as shown in Figure 3(b) can be expressed in the following equation:

$$
x=\frac{-B+\sqrt{B^{2}-4 A C}}{2 A},
$$

where

$$
\begin{aligned}
& A=\frac{\left(E_{c} b\right)}{\left(2 E_{f}\right)}, \\
& B=\frac{E_{s}\left(A_{s}+A_{s}^{\prime}\right)}{E_{f}+b_{f} d_{f}}, \\
& C=\frac{E_{s}\left(A_{s} d+A_{s}^{\prime} d^{\prime}\right)}{E_{f}+b_{f} d_{f} h_{f}} .
\end{aligned}
$$

Also, the cross section secondary moment obtained with the use of the cross section converted to fiber sheet may be expressed as follows:

$$
\begin{aligned}
I= & \frac{E_{c} b}{3 E_{f}} x^{3}+\frac{A^{\prime} E_{s}}{E_{f}}\left(x-d^{\prime}\right)^{2}+\frac{A_{s} E_{s}}{E_{f}}(d-x)^{2} \\
& +b_{f} d_{f}\left(h_{f}-x\right)^{2} .
\end{aligned}
$$

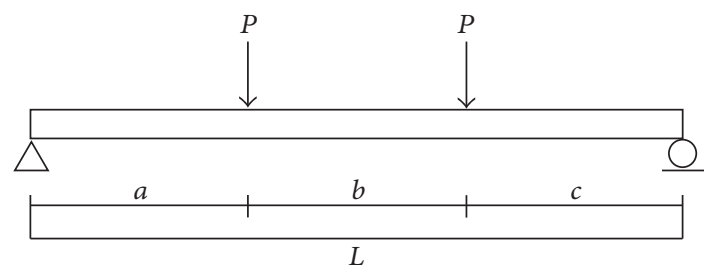

FIGURE 4: 2-point simply supported beam.

If $K_{s}, I$, and $x$ are substituted into the equation (13), end ripoff load; $P_{s}$ can be obtained [2]:

2.3. Deflection of Simply Supported Beam. The formula to calculate the deflection of the middle point of simply supported beam [14] is as shown in the following equation based on Figure 4:

$$
S_{\max }=\frac{P a}{2 E I}\left(\frac{2}{3} a^{2}+a b+\frac{1}{4} b^{2}\right) .
$$

Also, the formula to calculate the deflection of the part where load is applied is as follows:

$$
S=\frac{P a}{2 E I}\left(\frac{2}{3} a+b\right)
$$

where $E$ is Young's modulus $\left(\mathrm{kN} / \mathrm{cm}^{2}\right)$ and $I$ is moment of inertia $\left(\mathrm{cm}^{4}\right)$.

\section{Experiment Performance}

3.1. Experiment Plan. As for the RC beam used in the experiment, totally 4 beams were produced at length $2,400 \mathrm{~mm}$, width $150 \mathrm{~mm}$, height $250 \mathrm{~mm}$, and effective length $220 \mathrm{~mm}$. As for tensile rebar, deformed rebar D10 was used, and 

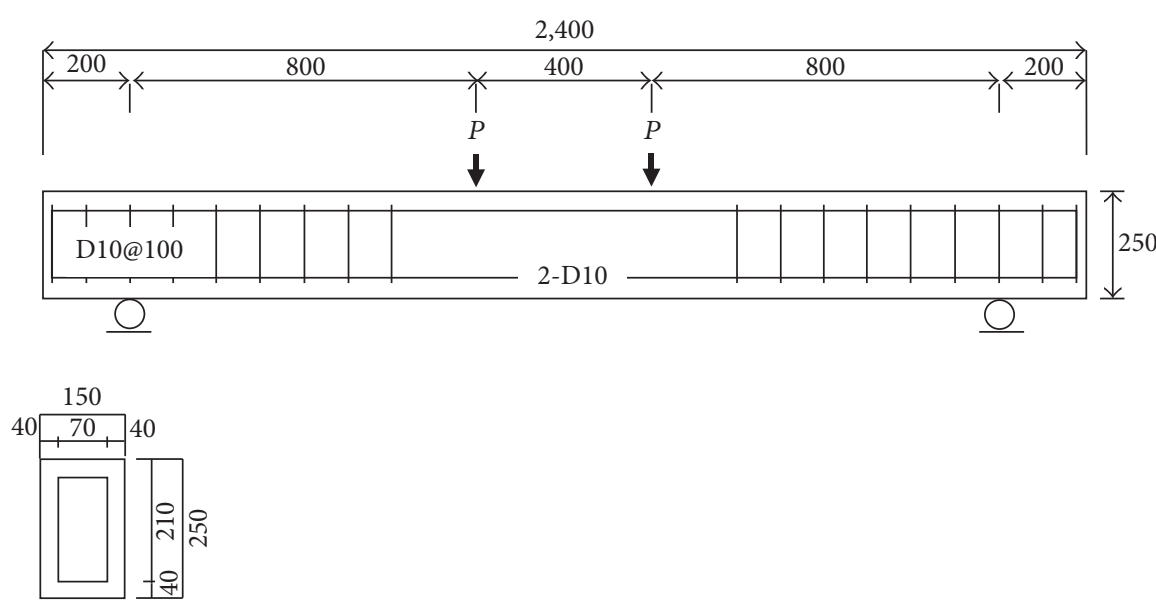

Figure 5: Specimen configuration.
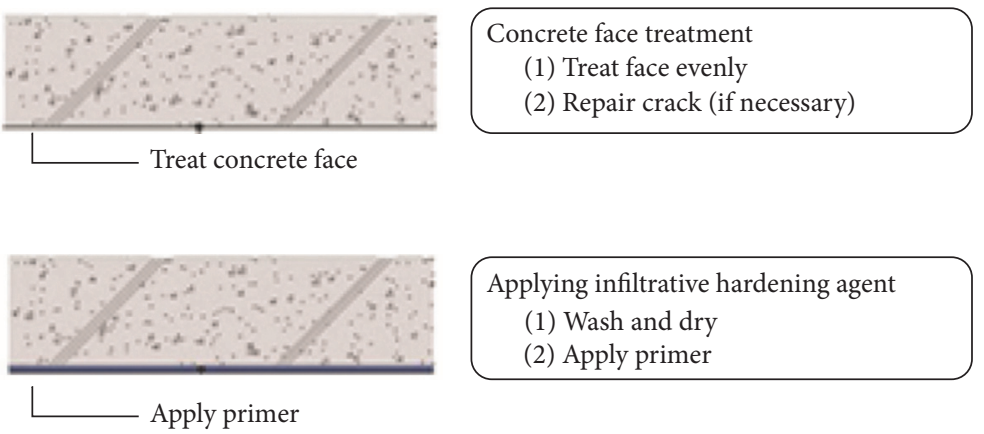

Applying infiltrative hardening agent

(1) Wash and dry

(2) Apply primer

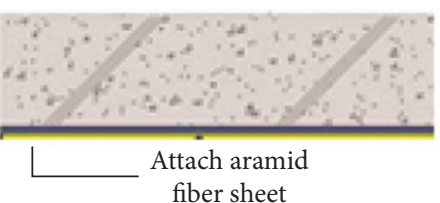

Applying infiltrative hardening agent

(1) Wash and dry

(2) Apply primer

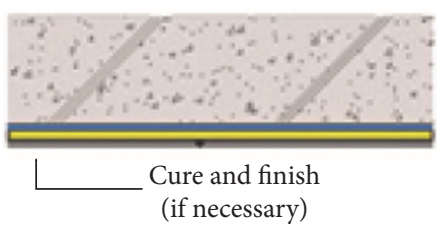

Adhere aramid fiber sheet
(1) Prepare adhesive
(2) Apply adhesive
(3) Perform work
(4) Attach sheet on the installed face

FIGURE 6: The procedure of installing aramid fiber sheets.

stirrups are as shown in Figure 5 where D10s are placed at $100 \mathrm{~mm}$ interval.

Concrete bending strength testing method by trisection point loading method was executed according to concrete flexural strength testing method [15].

The loads were applied gradually as concentrated load in displacement control method by being divided into trisection points with the use of 200 tons capacity actuator. The bending of beam was measured with the use of 4 LVDTs.

In order to examine the flexural performance of reinforcement material, aramid fiber sheets $(300 \mathrm{~mm}$ wide and $1.94 \mathrm{~mm}$ thick) were applied to the bottom part of beam, the bottom part and side part of beam, and the bottom part of beam and U-band both ends. The flexural performance of recycled aggregates RC beam reinforced with aramid fiber sheet varies significantly depending on the capacity of adhering aramid fiber sheet and RC beam. As shown in Figure 6, the installed parts of reinforced beam are cured by adhering aramid fiber sheet after treating the face and applying infiltrative hardening agent to the reinforced beam (see [16]).

The details of the 4 specimens are shown in Table 1, and the properties of each material [17] used are in Tables 2, 3, 4, and 5 . Recycled aggregates were used by being substituted by 
TABLE 1: The description of specimen group (H40-RCA30, H40RCA30-B, H40-RCA30-BU, and H40-RCA30-BS).

\begin{tabular}{lc}
\hline H40 & Design strength (MPa) \\
RCA30 & Recycled aggregates substitution rate (\%) \\
B & $\begin{array}{r}\text { Reinforcing method: bottom part reinforcement (B), } \\
\text { bottom + both end parts reinforcement (BU), bottom } \\
\text { + side part reinforcement (BS) }\end{array}$ \\
\hline
\end{tabular}

TABle 2: Properties of materials.

\begin{tabular}{lcc}
\hline Materials & \multicolumn{2}{c}{ Properties } \\
\hline \multirow{2}{*}{ Concrete } & Compressive strength (MPa) & 41.13 \\
& Modulus of elasticity (Gpa) & 29.40 \\
\hline & Tensile strength (Mpa) & 2059.43 \\
Aramid fiber (Sheet 5) & Modulus of elasticity (Gpa) & 117.68 \\
& Proof stress (ton) & 12 \\
& Design thickness (mm) & 1.94 \\
\hline
\end{tabular}

TABLE 3: The characteristics and properties of adhesives for aramid fiber sheets.

\begin{tabular}{lccc}
\hline $\begin{array}{l}\text { Product } \\
\text { name }\end{array}$ & Property & $\begin{array}{c}\text { Reference } \\
\text { value }\left(\mathrm{kg} / \mathrm{cm}^{2}\right)\end{array}$ & Testing method \\
\hline SK-CPA105 & $\begin{array}{c}\text { Compressive } \\
\text { strength } \\
\text { Tensile strength } \\
\text { Adhesive } \\
\text { strength } \\
\text { Concrete }\end{array}$ & 910 or above & ASTM D695 \\
& 100 above & ASTM D1002 \\
adhesive strength & 15 or above & JIS K5400 \\
\hline
\end{tabular}

TABLE 4: The properties of recycled aggregates.

\begin{tabular}{lcc}
\hline \multirow{2}{*}{ Physical property } & \multicolumn{2}{c}{ Recycled coarse aggregates } \\
& KS F 2573 & Measured value \\
\hline Absorption rate (\%) & Type 1 & 1.67 \\
& $\leq 3.0$ & \\
Density $\left(\mathrm{g} / \mathrm{cm}^{2}\right)$ & $\geq 2.5$ & 2.57 \\
Abrasion reduction & $\leq 40$ & 22.18 \\
0.08 mm sieve passing amount & $\leq 1.0$ & 0.89 \\
\hline
\end{tabular}

$30 \%$ based on Table 2 according to the standard [18], and mix proportion table is as shown in Table 5.

Figure 7 shows the average compressive strength of concrete 2 cylinder specimens of each member based on the standard of KS F 2403.

\section{Experiment Result}

4.1. Final Failure Mode. The final failure of specimen went through the process of reaching final failure as fiber sheets break or rip off after initial lateral crack occurs, and the final

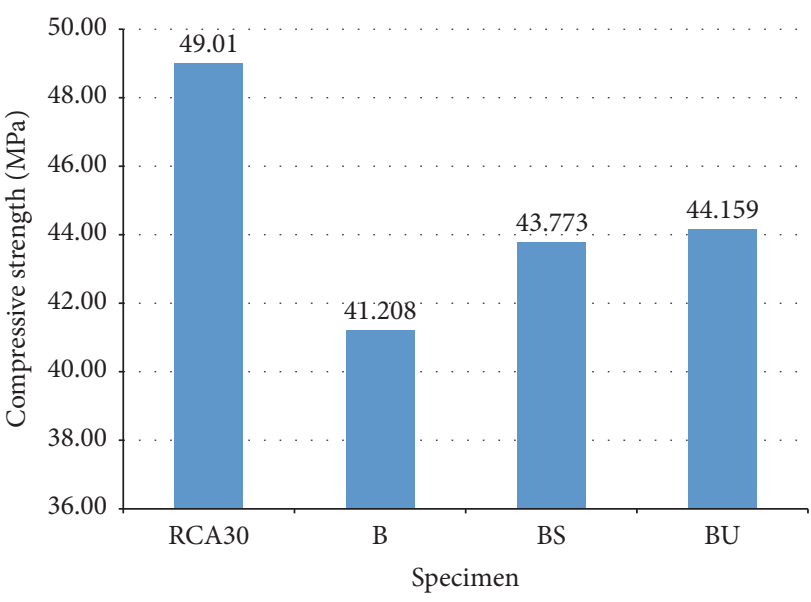

FIGURE 7: Compressive strength test result of specimen.

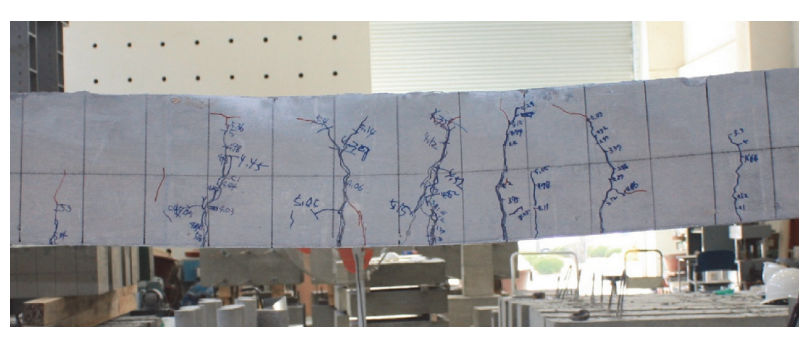

Figure 8: Specimen failure (H40-RCA30).

failure mode (shape) by specimen can be summed up as follows.

(1) The Reference Specimen of H40-RCA30. As for reference specimen H40-RCA30 as shown in Figure 8, initial lateral crack occurred at the central part at 2.12 tonf of load, and at load 3.57 tonf, crack occurred at diagonal tension bar. As the load increased, diagonal tension bar crack progressed from member bottom part toward actuating point, and as the bending crack at the center moves on, the width further increased. The specimen finally broke off as the crack width increased without any crack occurring until maximum 6.42 tonf after 5.04 tonf load.

(2) H40-RCA30-B. In H40-RCA30-B specimen as shown in Figure 9 which is reinforced with aramid fiber sheet at the bottom part, diagonal tension bar crack occurred at the bottom part on the right side at load 3.98 tonf. At load 7.04 tonf, the gauge broke off; displacement of $18 \mathrm{~mm}$ occurred at load of 8.29 tonf, and part of the aramid broke off. As the aramid fiber sheet at load 8.98 tonf ripped off and split apart and bending crack width increased, bottom housing concrete broke off.

(3) H40-RCA30-BU. In the H40-RCA30-BU specimen, as shown in Figure 10, of which bottom part and both end parts were reinforced, diagonal tension bar crack occurred at load 3.85 tonf, and at load 7.3 tonf, the rip-off of aramid fiber sheet sounded. As rip-off of aramid fiber sheet progressed fast 
TABLE 5: Mix proportion of concrete specimen.

\begin{tabular}{lccccccc}
\hline \multirow{2}{*}{ Design strength (Mpa) } & & \multicolumn{4}{c}{ Weight of material $\left(\mathrm{kg} / \mathrm{m}^{3}\right)$} \\
& Water & Cement & Blast-furnace slag & Sand & Natural aggregate & Recycled aggregate & AE agent \\
\hline 40 & 167 & 328 & 141 & 850 & 595 & 261 & 5.16 \\
\hline
\end{tabular}
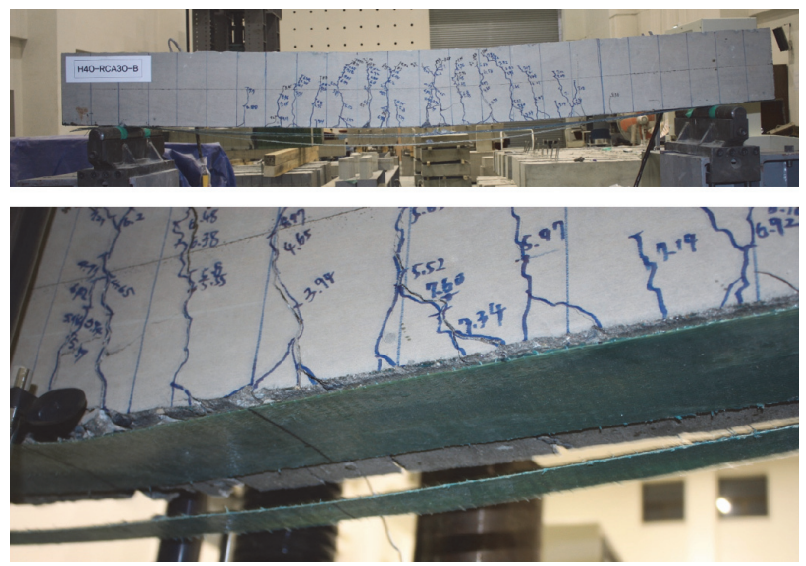

FIGURE 9: Specimen failure pattern (H40-RCA30-B).
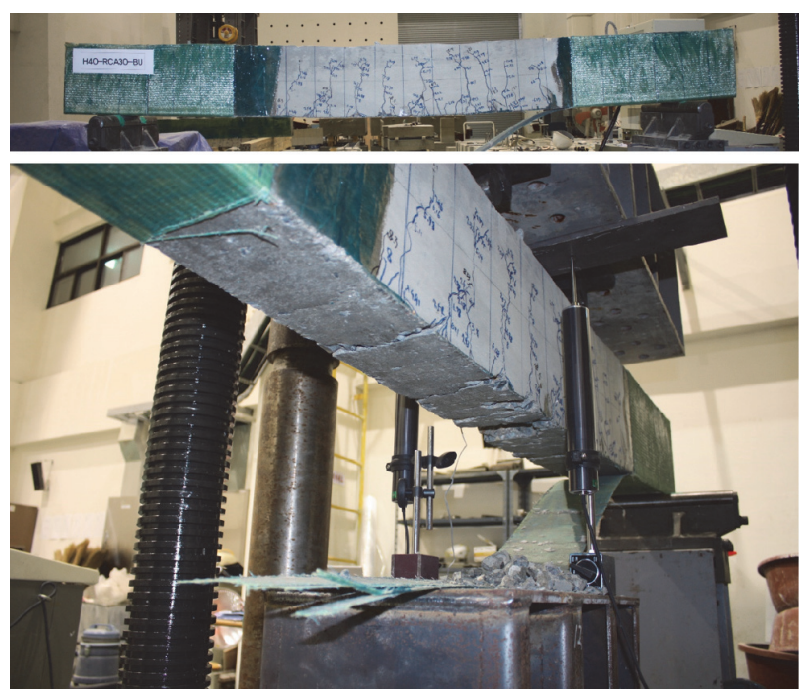

FIGURE 10: Specimen failure mode (H40-RCA30-BU).

at load 8.35 tonf, numerous cracks occurred. As the bottom aramid fiber sheet at the end broke off at load 8.86 tonf, the sheet finally broke off.

(4) H40-RCA30-BS. As for H40-RCA30-BS, as shown in Figure 11, of which bottom part and side part were reinforced, no crack was found due to reinforcing agent. At load 11.15 tonf, the rip-off of aramid fiber sheet at the location began to sound, but no bending occurred at all. As the adhesion of aramid fiber sheet and specimen failed due to excessive deformation of specimen at load 13.45 tonf, the specimen finally broke off.
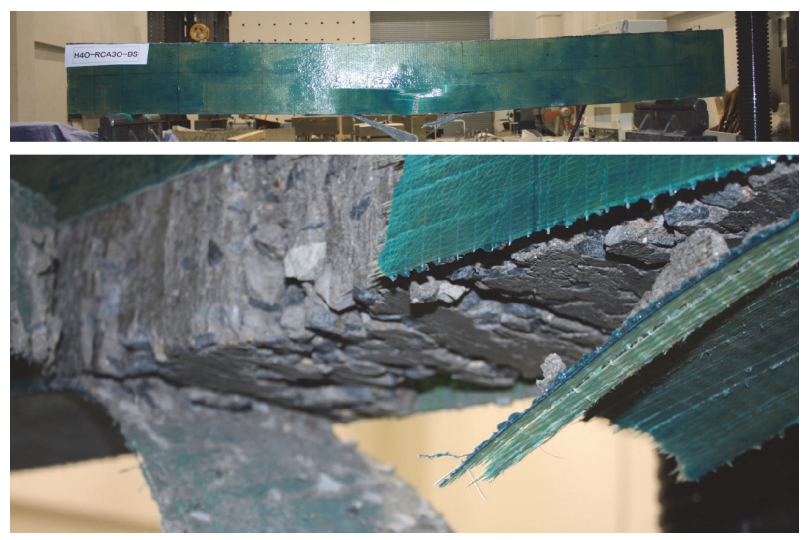

FIGURE 11: Specimen failure pattern (H40-RCA30-BS).

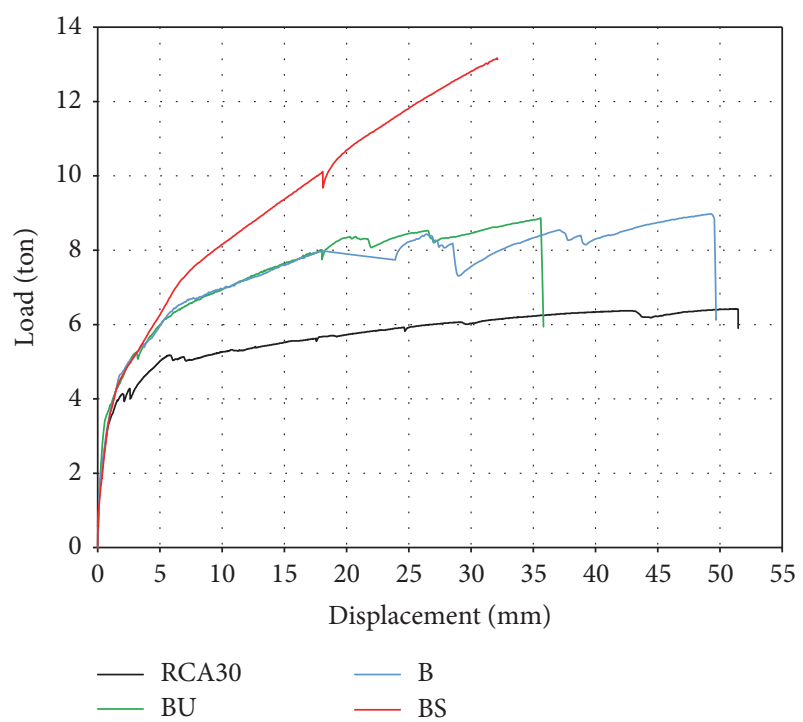

Figure 12: Load-displacement curve.

4.2. Maximum Load. Table 6 shows the load at which initial crack occurred to unreinforced and reinforced specimens, yield load, maximum load, and displacement for each load. Also, Tables 7, 8, and 9 show the comparison of the theoretical values of the tensile failure, rip-off breaking load, and maximum displacement of H40-RCA30-B and the actual values thereof, and the load-displacement curve is shown in Figure 12.

It is shown that reinforced specimen has increased in yield load and maximum resistance compared with unreinforced specimen, and the increased degree differs depending on attaching method. Reinforced specimen decreases in load rapidly after reaching maximum resistance, remaining at 
TABLE 6: Experiment result.

\begin{tabular}{lcccccc}
\hline \multirow{2}{*}{ Specimen name } & \multicolumn{2}{c}{ Initial crack } & \multicolumn{2}{c}{ At yielding } & \multicolumn{2}{c}{ At maximum resistance } \\
& Load (ton) & Displacement $(\mathrm{mm})$ & Load (ton) & Displacement (mm) & Load (ton) & Displacement (mm) \\
\hline H40-RCA30 & 2.12 & 0.36 & 5.03 & 7.18 & 6.42 & 51.39 \\
H40-RCA30-B & 3.94 & 1.26 & 6.71 & 7.94 & 7.98 & 49.24 \\
H40-RCA30-BU & 3.85 & 1.20 & 6.56 & 7.395 & 12.05 & 13.45 \\
H40-RCA30-BS & $\mathrm{x}$ & $\mathrm{x}$ & 8.66 & & 35.61 & 33.08 \\
\hline
\end{tabular}

TABLE 7: Comparative analysis of H40-RCA30-B.

\begin{tabular}{|c|c|c|c|}
\hline Specimen name & $\begin{array}{l}\text { Theoretical value of tensile failure load } \\
\text { (ton) }\end{array}$ & $\begin{array}{l}\text { Theoretical value of end rip-off load } \\
\text { (ton) }\end{array}$ & Experimental maximum load (ton) \\
\hline H40-RCA30-B & 10.14119 & 8.54671 & 8.98 \\
\hline
\end{tabular}

TABLE 8: Comparative analysis of H40-RCA30-B.

\begin{tabular}{lcc}
\hline Specimen name & $\begin{array}{c}\text { Theoretical maximum } \\
\text { displacement }(\mathrm{mm})\end{array}$ & $\begin{array}{c}\text { Experimental maximum } \\
\text { displacement }(\mathrm{mm})\end{array}$ \\
\hline H40-RCA30-B & 39.56 & 49.24 \\
\hline
\end{tabular}

TABLE 9: Comparison of ductility.

\begin{tabular}{lcc}
\hline Specimen name & Stiffness (ton/mm) & Ductility ratio \\
\hline H40-RCA30 & 0.700557 & 7.157382 \\
H40-RCA30-B & 0.845088 & 6.200882 \\
H40-RCA30-BU & 0.887086 & 4.815416 \\
H40-RCA30-BS & 0.718672 & 2.745228 \\
\hline
\end{tabular}

similar level to unreinforced specimen, and the behavior thereafter also is similar to unreinforced specimen.

4.3. Evaluation of Initial Stiffness and Ductility. The rigidity of specimen was obtained by dividing the yield load, $80 \%$ of maximum load, by the displacement at yield load. Also, ductility ratio was obtained by dividing the displacement at maximum load by the displacement at yield load as shown in Figure 13.

\section{Conclusion}

This study has the purpose of developing sustainable structural concrete by evaluating the reinforced effects of RC beam strengthened by aramid fiber sheet to which recycled aggregates were substituted by $30 \%$ and through the analysis of the load-displacement curve, yield load, maximum load, ductility ratio, concrete crack, and fiber sheet failure pattern obtained from the experiment. The following conclusions were reached as the result of study:

(1) Compared with unreinforced existing specimen, the load of specimen at which initial crack occurred increased by $85.8 \%$ in case of H40-RGA30-B and by $79.2 \%$ in case of H40-RGA30-BU, so it is judged that RCB aramid fiber sheet would provide effective reinforcement.

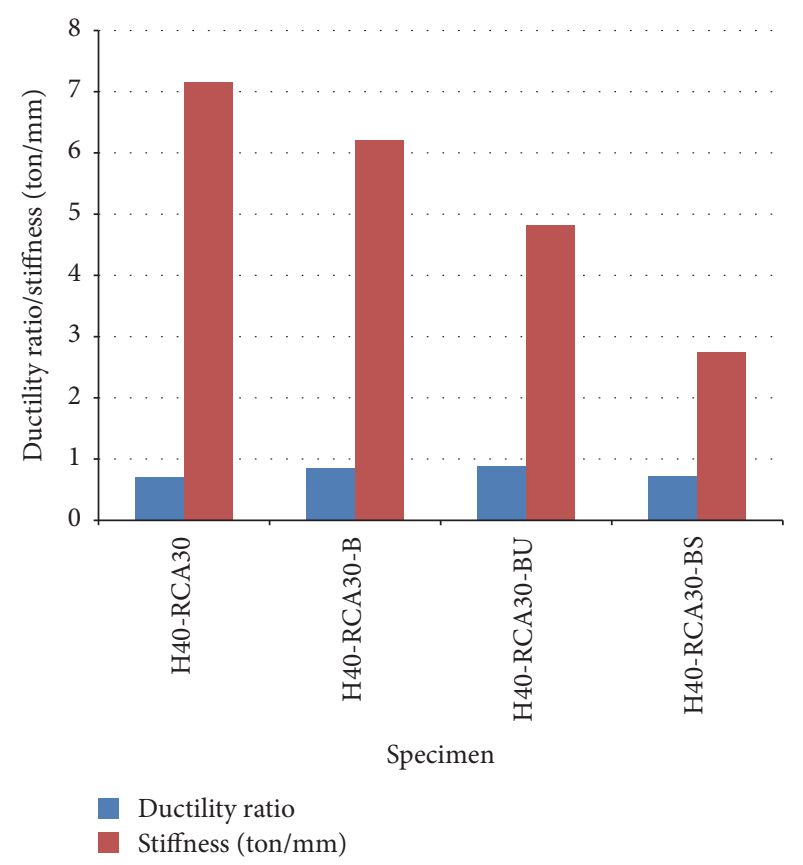

FIGURE 13: Comparison of rigidity ratio and ductility ratio by specimen.

(2) Compared with existing specimen, the crack occurring interval and width, and so forth of specimen reinforced with aramid fiber sheet were found to be narrower. This is judged to exist because loads were delivered evenly due to the effect of aramid fiber sheet.

(3) As for ultimate loads, H40-RCA30-B increased by $38.01 \%, \mathrm{H} 40-\mathrm{RCA} 30-\mathrm{BU}$ by $39.88 \%$, and $\mathrm{H} 40$ RGA30-BS by $100.79 \%$. From this, it is judged that RCB aramid fiber sheet offers reinforcing effects.

(4) Ductility ratios were 6.20 for H40-RGA30-B, 4.82 for H40-RGA30-BU, and 2.75 for H40-RGA30-BS, so it is judged that H40-RGA30-B has outstanding ductile capacity. 


\section{Competing Interests}

The authors declare that they have no competing interests.

\section{Acknowledgments}

This research was supported by Basic Science Research Program through the National Research Foundation of Korea (NRF) funded by the Ministry of Education Science and Technology (KRF-2010-0025651) and supported under the framework of international cooperation program managed by National Research Foundation of Korea (2011-0030929).

\section{References}

[1] M. Shahria Alam, E. Slater, and A. H. M. Muntasir Billah, "Green concrete made with RCA and FRP scrap aggregate: fresh and hardened properties," Journal of Materials in Civil Engineering, vol. 25, no. 12, pp. 1783-1794, 2013.

[2] H. H. Lee, E. S. Koo, and L. H. Lee, "A comparative study on the flexural capacity of the beam with carbon fiber sheet and aramid fiber sheet," Journal of Architectural Institute of Korea, Structural Field, vol. 14, no. 9, pp. 65-72, 1998.

[3] J. De Brito, J. Ferreira, J. Pacheco, D. Soares, and M. Guerreiro, "Structural, material, mechanical and durability properties and behaviour of recycled aggregates concrete," Journal of Building Engineering, vol. 6, pp. 1-16, 2016.

[4] K. El-Gamil Hassan, J. M. Reid, and M. S. Al-Kuwari, "Recycled aggregates in structural concrete-a Qatar case study," Proceedings of the Institution of Civil Engineers-Construction Materials, vol. 169 , no. 2, pp. 72-82, 2016.

[5] R. Jin, Q. Chen, and A. Soboyejo, "Survey of the current status of sustainable concrete production in the U.S.," Resources, Conservation and Recycling, vol. 105, pp. 148-159, 2015.

[6] S. D. Kim, B. S. Cho, and J. W. Seong, "Flexural behaviors of reinforced concrete beams strengthened with glass fiber sheets," Journal of Korean Society for Railway, vol. 12, no. 3, pp. 388-395, 2009.

[7] G. M. Chen, J. G. Teng, and J. F. Chen, "Process of debonding in RC beams shear-strengthened with FRP U-strips or side strips," International Journal of Solids and Structures, vol. 49, no. 10, pp. 1266-1282, 2012.

[8] R. T. Erdem, M. Bağcl, and A. Demir, "A comparative evaluation of performance based analysis procedures according to 2007 Turkish Earthquake Code and FEMA-440," Mathematical and Computational Applications, vol. 16, no. 3, pp. 605-616, 2011.

[9] M. Saafi and H. Toutanji, "Flexural capacity of prestressed concrete beams reinforced with aramid fiber reinforced polymer (AFRP) rectangular tendons," Construction and Building Materials, vol. 12, no. 5, pp. 245-249, 1998.

[10] M. A. Pisani, "Long-term behaviour of beams prestressed with aramid fibre cables: part 1: a general method," Engineering Structures, vol. 22, no. 12, pp. 1641-1650, 2000.

[11] H. H. Lee, E. S. Koo, and L. H. Lee, "An experimental study on the behavior of RC beams strengthened with carbon fiber sheets," Journal of Architectural Institute of Korea, Structural Field, vol. 14, no. 6, pp. 77-88, 1998.

[12] A. Brückner, R. Ortlepp, and M. Curbach, “Textile reinforced concrete for strengthening in bending and shear," Materials and Structures, vol. 39, no. 292, pp. 741-748, 2006.
[13] T. M. Roberts, "Approximate analysis of shear and normal stress concentrations in the adhesive layer of plated RC beams," Structural Engineer, vol. 67, no. 12, pp. 228-233, 1989.

[14] H. N. Cho, Structural Mechanics, Gumi Book Publisher, 1995.

[15] Korea Standard Association, F 2408: Concrete Bending Strength Testing Method, Industrial Standard Screening Committee, Korea Standard Association, Seoul, Republic of Korea, 2000.

[16] Heesang Reinforce, http://heesang.co.kr/.

[17] KS (Korean Standard), F 2403: How to produce specimen for concrete strength test, Ministry of Knowledge Economy Agency for Technology and Standard, Seoul, Korea, 2010.

[18] KS (Korean Standard), F 2573 Recycled Aggregates for Concrete, Ministry of Knowledge Economy Agency for Technology and Standard, Seoul, Korea, 2011. 

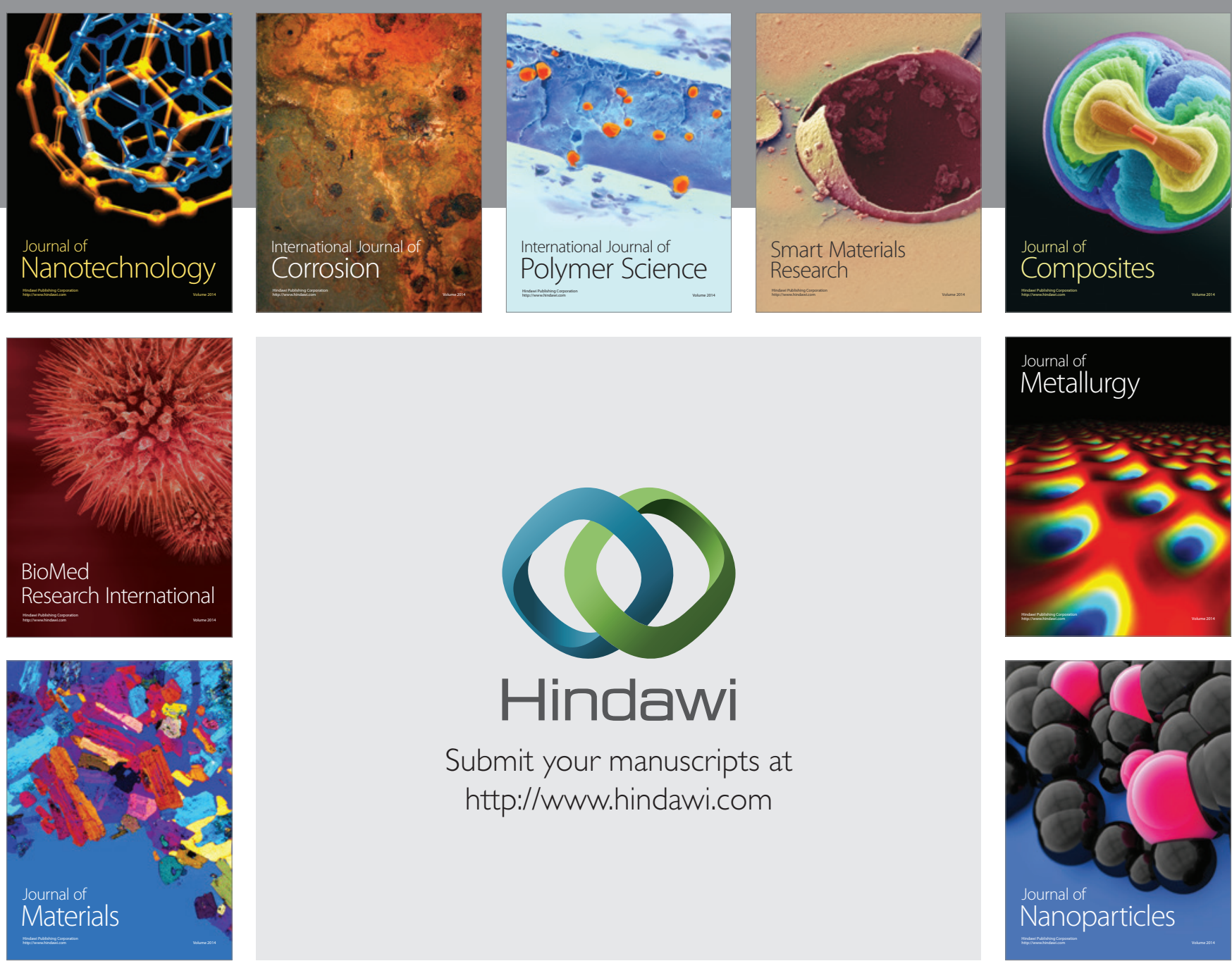

\section{Hindawi}

Submit your manuscripts at

http://www.hindawi.com

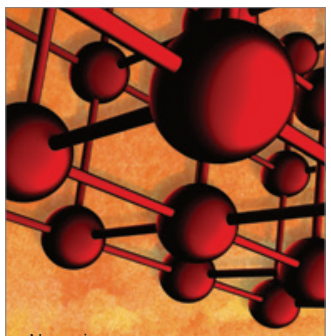

Materials Science and Engineering
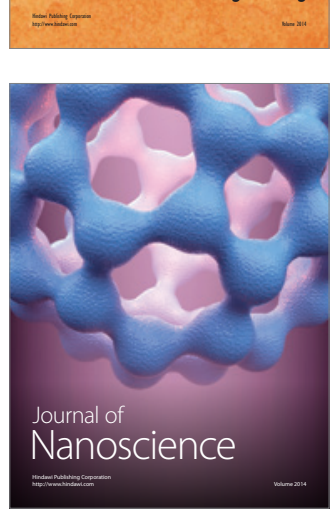
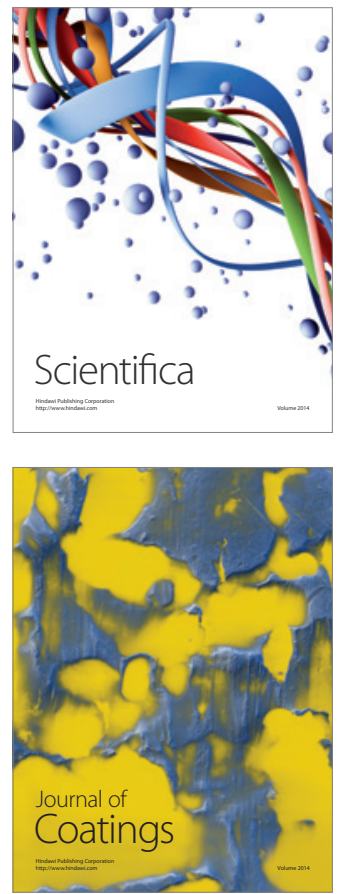
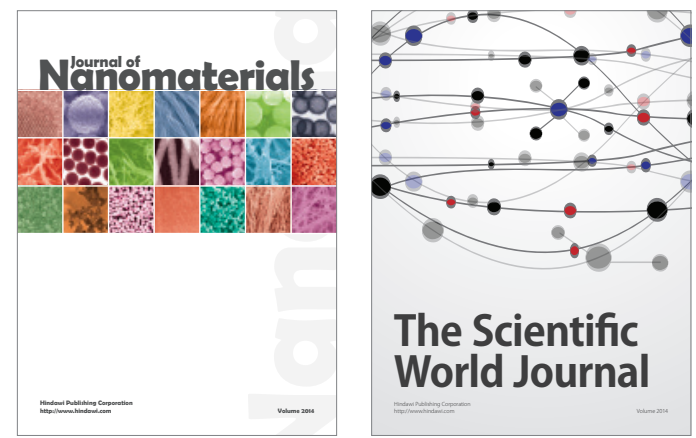

The Scientific World Journal
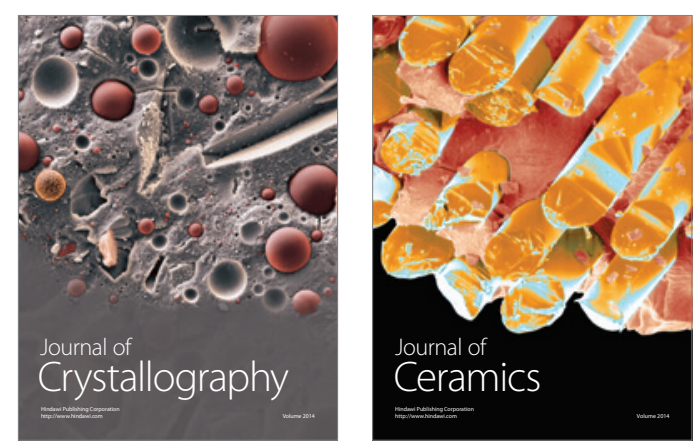
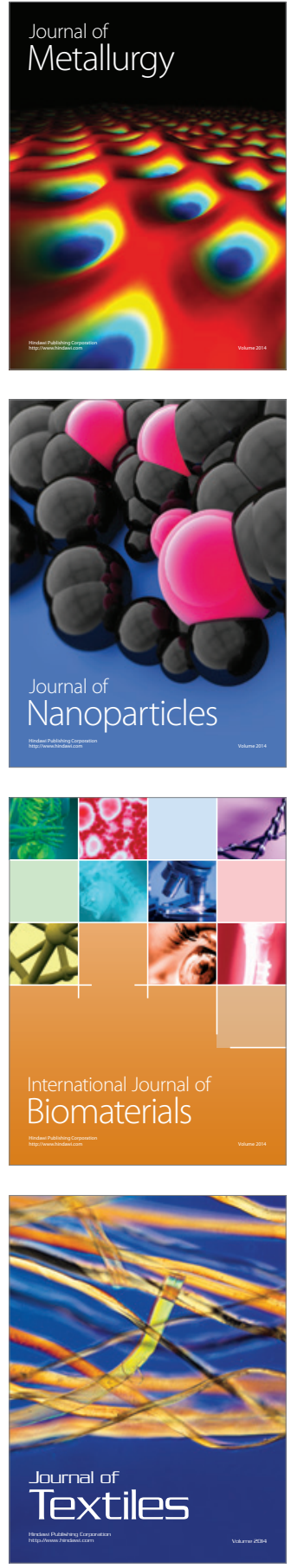